(14) have demonstrated that the effects of MDA-LDL immunization are associated with increased titers of the IgM-antibody isotype. However, future studies will need to determine the relative contribution of T15/ EO6 IgMs to the reduction in atherosclerosis versus that of other IgMs that are formed during MDA-LDL immunization. Finally, it will be important to determine whether the T15/EO6-mediated inhibition of OxLDL uptake by macrophages observed in cultured cells is the mechanism responsible for the reduced atherosclerotic lesion size.

Overall, these studies have mapped out an interesting and novel pathway linking the adaptive and natural arms of the immune system (14). These findings also highlight a practical issue that needs to be considered for future therapeutic strategies. Specifically, antagonists of IL-5 are being developed for asthma therapy. The studies of Binder et al. indicate that it will be important to determine the ramifications of such a therapy on vascular disease.

Address correspondence to: Alan Daugherty, Wethington Building, Room 521, 900 S. Limestone Road, University of Kentucky, Lexington, Kentucky 40536-0200, USA. Phone: (859) 323-4933 ext. 81389; Fax: (859) 257-3646; E-mail: Alan.Daugherty@uky.edu.
1. Henriksen, T., Mahoney, E.M., and Steinberg, D. 1981. Enhanced macrophage degradation of low density lipoprotein previously incubated with cultured endothelial cells: Recognition by receptors for acetylated low density lipoproteins. Proc. Natl. Acad. Sci. U. S. A. 78:6499-6503.

2. Steinberg, D., Parthasarathy, S., Carew, T.E., Khoo, J.C., and Witztum, J.L. 1989. Beyond cholesterol. Modifications of low density lipoprotein that increase its atherogenicity. N. Engl.J. Med. 320:915-924.

3. Stemme, S., et al. 1995. T lymphocytes from human atherosclerotic plaques recognize oxidized low density lipoprotein. Proc. Natl. Acad. Sci. U. S. A. 92:3893-3897.

4. Palinski, W., et al. 1989. Low density lipoprotein undergoes oxidative modification in vivo. Proc. Natl. Acad. Sci. U. S. A. 86:1372-1376.

5. Salonen, J.T., et al. 1992. Autoantibody against oxidised LDL and progression of carotid atherosclerosis. Lancet. 339:883-887.

6. Palinski, W., Miller, E., and Witztum, J.L. 1995. Immunization of low density lipoprotein (LDL) receptordeficient rabbits with homologous malondialdehydemodified LDL reduces atherogenesis. Proc. Natl. Acad. Sci.U.S. A. 92:821-825

7. Ameli, S., et al. 1996. Effect of immunization with homologous LDL and oxidized LDL on early atherosclerosis in hypercholesterolemic rabbits. Arterioscler. Thromb. Vasc. Biol. 16:1074-1079.

8. Freigang, S., Horkko, S., Miller, E., Witztum, J.L., and Palinski, W. 1998. Immunization of LDL receptor-deficient mice with homologous malondialdehyde-modified and native LDL reduces progression of atherosclerosis by mechanisms other than induction of high titers of antibodies to oxidative neoepitopes. Arterioscler. Thromb. Vasc. Biol. 18:1972-1982.

9. Palinski, W., et al. 1994. ApoE-deficient mice are a model of lipoprotein oxidation in atherogenesis - Demonstration of oxidation-specific epitopes in lesions and high titers of autoantibodies to malondialdehyde-lysine in serum. Arterioscler. Thromb. 14:605-616.

10. Palinski, W., et al. 1996. Cloning of monoclonal autoantibodies to epitopes of oxidized lipoproteins from apolipoprotein E-deficient mice - Demonstration of epitopes of oxidized low density lipoprotein in human plasma. J. Clin. Invest. 98:800-814.

11. Horkko, S., et al. 1999. Monoclonal autoantibodies specific for oxidized phospholipids or oxidized phospholipid-protein adducts inhibit macrophage uptake of oxidized low-density lipoproteins. J. Clin. Invest. 103:117-128.

12. Shaw, P.X., et al. 2000. Natural antibodies with the T15 idiotype may act in atherosclerosis, apoptotic clearance, and protective immunity. J. Clin. Invest. 105:1731-1740.

13. Binder, C.J., et al. 2003. Pneumococcal vaccination decreases atherosclerotic lesion formation: molecular mimicry between Streptococcus pneumoniae and oxidized LDL. Nat. Med. 9:736-743.

14. Binder, C.J., et al. 2004. IL-5 links adaptive and natural immunity specific for epitopes of oxidized LDL and protects from atherosclerosis. J. Clin. Invest. 114:427-437. doi:10.1172/JCI200420479.

15. Zhou, X.H., Paulsson, G., Stemme, S., and Hansson, G.K. 1998. Hypercholesterolemia is associated with a $\mathrm{T}$ helper (Th) $1 / \mathrm{Th} 2$ switch of the autoimmune response in atherosclerotic apoE-knockout mice. J. Clin. Invest. 101:1717-1725.

16. Pinderski, L.J., et al. 2002. Overexpression of interleukin-10 by activated $\mathrm{T}$ lymphocytes inhibits atherosclerosis in LDL receptor-deficient mice by altering lymphocyte and macrophage phenotypes. Circ. Res. 90:1064-1071.

17. Pinderski-Oslund, L.J., et al. 1999. Interleukin-10 blocks atherosclerotic events in vitro and in vivo. Arterioscler. Thromb. Vasc. Biol. 19:2847-2853.

18. Mallat, Z., et al. 1999. Protective role of interleukin10 in atherosclerosis. Circ. Res. 85:E17-E24.

\title{
Pulmonary fibrosis: thinking outside of the lung
}

\author{
Stavros Garantziotis, ${ }^{1}$ Mark P. Steele, ${ }^{1}$ and David A. Schwartz ${ }^{1,2}$
}

${ }^{1}$ Department of Medicine at Duke University Medical Center; ${ }^{2}$ Durham Department of Veterans Affairs Medical Center, Durham, North Carolina, USA.

\begin{abstract}
Pulmonary fibrosis is a devastating condition that leads to progressive lung destruction and scarring. Previous mechanistic research has focused on the local fibroproliferative process in the lung. However, emerging evidence suggests that circulating cells of hematopoietic origin play a crucial role in the pathogenesis of this disease (see the related article beginning on page 438).
\end{abstract}

Idiopathic interstitial pneumonias (IIPs; see Table 1) are a group of devastating conditions isolated to the lung and, except by lung transplantation, often are untreatable. While idiopathic pulmonary fibrosis (IPF) is the most common type of IIP and involves dense fibrosis with active areas of

Nonstandard abbreviations used: CXC chemokine ligand 12 (CXCL12); idiopathic interstitial pneumonia (IIP); idiopathic pulmonary fibrosis (IPF).

Conflict of interest: D.A. Schwartz serves as a scientific advisor for Intermune.

Citation for this article: J. Clin. Invest. 114:319-321 (2004). doi:10.1172/JCI200422497. fibroproliferation, other forms of IIP are characterized by varying degrees of inflammation in addition to the active fibroproliferative process. Although pulmonary fibrosis has traditionally been thought to develop in response to chronic inflammation in the lung parenchyma, emerging evidence suggests that repeated exogenous and endogenous stimuli cause sequential lung injury that results in pulmonary fibrosis in individuals unable to effectively heal the damage (Figure 1) (1).

The key to understanding the complex pathogenesis of IIP may lie in the answers to two very simple questions: why is this condition isolated to the lung, and do factors outside of the lung affect the pathogenesis of this disease?

\section{Circulating fibrocytes and pulmonary fibrosis}

In this issue of the JCI, Phillips and colleagues (2) endeavor to answer these questions by identifying the circulating fibrocyte and chemokine gradients critical to the fibroproliferative process in a murine model of bleomycin-induced pulmonary fibrosis. Fibrocytes are a unique population of circulating cells of hematopoietic origin that produce ECM proteins (e.g., fibroblast-like collagen and vimentin and myofibroblast-like $\alpha$-smooth muscle actin) and differentiate into myofibroblasts (3). These cells are characterized by specific 
Table 1

IIPs and their relative frequency

\begin{tabular}{ll} 
Type of IIP & Frequency \\
Idiopathic pulmonary fibrosis & $55 \%$ \\
Nonspecific interstitial pneumonia & $25 \%$ \\
Acute interstitial pneumonia & $<1 \%$ \\
Cryptogenic organizing pneumonia & $3 \%$ \\
Respiratory bronchiolitis-interstitial lung disease and & $15 \%$ \\
$\quad$ desquamative interstitial pneumonia & \\
Lymphocytic interstitial pneumonia & $<1 \%$ \\
\hline
\end{tabular}

The information in this table is derived from refs. 11 and 19.

cell surface markers (CD34, CD11b, CD18, CD45, and HLA-DR) and the expression of several chemokine receptors (CCR3, CCR5, CCR7, and CXCR4) (3). Fibrocytes have been identified in the expanding margins of wounds (4), scleroderma skin biopsies (5), and bronchial walls in asthmatics (6). In a series of elegant experiments, Phillips et al. have discovered that the fibrocyte also plays an important role in the development of pulmonary fibrosis following bleomycin-induced injury in mice. Their findings demonstrate that: (a) human fibrocytes chemotax to the mouse lung in response to $\mathrm{CXC}$ chemokine ligand 12 (CXCL12); (b) human fibrocytes localize to the lung following bleomycin-induced lung injury in mice; and (c) systemic administration of anti-CXCL12 antibody reduces the accumulation of lung fibrocytes and collagen as well as, according to histologic evidence, pulmonary fibrosis following administration of bleomycin to mice. Importantly, the investigators also demonstrate that bleomycin administration causes proliferation of bone marrow fibrocytes, which suggests that this pool is

\section{Figure 1}

Proposed mechanism for the pathogenesis of pulmonary fibrosis. The lung is naturally exposed to repetitive injury from a variety of exogenous and endogenous stimuli. Several local and systemic factors (e.g., fibroblasts, circulating fibrocytes, chemokines, growth factors, and clotting factors) contribute to tissue healing and functional recovery. Dysregulation of this intricate network through genetic predisposition, autoimmune conditions, or superimposed diseases can lead to aberrant wound healing with the result of pulmonary fibrosis. Alternatively, excessive injury to the lung may overwhelm even intact reparative mechanisms and lead to pulmonary fibrosis. The figure was adapted with permission from the New England Journal of Medicine (1). the likely source of fibrocytes that eventually aggregate in the lung.

While Phillips and colleagues are believed to be the first to find that systemic circulating fibrocytes are involved in the pathogenesis of lung fibrosis and that CXCL12 (or possibly CXCR4 ligands in general) play an important role in the trafficking of these cells to the lung (2), others have recently found that bone marrow-derived cells also appear to be critical to the fibroproliferative response in the lung (7). Together, these investigative groups provide evidence to support novel mechanisms underlying the pathogenesis of pulmonary fibrosis.

\section{Implications for pulmonary fibrosis}

If indeed circulating multipotent cells respond to the fundamental pathogenic signals of lung injury by actively migrating to the primary wound, and if these hematopoietic cells play a role in the fibroproliferative response that ultimately leads to pulmonary fibrosis, then the primary injurious events that contribute to the homing signals in the lung, the source of these cells, and the factors that facilitate the proliferation of these cells need to be carefully considered. In fact, the fibroproliferative and inflammatory processes in the lung may be too far downstream from the primary events to substantially contribute to the pathophysiological basis of pulmonary fibrosis. The more proximal events (microscopic lung injury, homing signals, and factors that induce the proliferation of multipotent hematopoietic cells) may provide critical clues to understanding this biologically complex process.

There are many potential stimuli that injure the lung and set the stage for the development of IIP. As approximately 11,000 liters of air move through the lung each day, one can argue that all of us, by virtue of living in a world with dusts, smoke, and aerosolized toxins and microorganisms, experience recurrent episodes of lung injury that require repair and regeneration of normal lung tissue. Epidemiological evidence suggests that such exposure contributes to the development of pulmonary fibrosis: IPF occurs more frequently in males (8), cigarette smokers (9), and individuals that experience occupational exposure to metal or wood dust (10). Moreover, occupational exposure to asbestos can cause pulmonary fibrosis that is indistinguishable from IPF, and cytotoxic drugs and autoimmune conditions (e.g., collagen vascular diseases and inflammatory bowel diseases) can lead to other forms of IIP (11). In aggregate, the findings of Phillips et al. suggest that both exogenous and endogenous factors can provide the primary stimuli that lead to lung injury and focus the reparative process in the lung.

While structural lung cells could facilitate this repair, terminal differentiation of these 
cells may limit their plasticity. Teleologically, it makes much more sense that circulating cells and even bone marrow-derived multipotent cells are the fundamental biological elements called upon to rejuvenate the injured lung. Although Phillips and colleagues (2) have highlighted the importance of the circulating fibrocyte and the chemokine activity of CXCL12, there are undoubtedly other cells and molecules that induce the fibrotic response in the lung and ultimately lead to progressive tissue destruction and abnormal gas exchange. In fact, Phillips and his colleagues have shown that, even after treatment with neutralizing CXCL12 antibody, bleomycin can cause lung fibrosis and fibrocyte recruitment (2), which indicates that additional mechanisms are surely involved in the development of pulmonary fibrosis.

But then why do only some individuals go on to develop pulmonary fibrosis? From a simplistic perspective, one could reason that pulmonary fibrosis develops as a result of excessive lung injury, insufficient repair mechanisms, or a combination of these two events. While we know that excessive lung injury can result in pulmonary fibrosis (e.g., asbestosis), considerable variability in the extent of pulmonary fibrosis exists even among those individuals exposed to similar concentrations of this fibrogenic agent (12). In fact, the genetic background underlying the propensity for fibrosis can hardly be overlooked: pulmonary fibrosis clusters in families (13) and occurs in pleiotropic genetic disorders (14), and inbred strains of mice demonstrate variable susceptibility to fibrogenic agents (15-17). Some hosts, perhaps by virtue of defective repair mechanisms, simply are unable to deal with a fibrogenic insult.

The work of Phillips and colleagues (2) raises several important unanswered questions about the mechanisms and regulation of fibrocyte migration to the lung and the role of these cells in pulmonary fibrosis. Specifically, what is the circulating signal(s) from the injured lung that reaches the bone marrow to stimulate recruitment? Once fibrocytes migrate to the lung, what is their fate? Do the fibrocytes persist indefinitely, migrate out of the lung, or undergo apoptosis? Are the fibrocytes proliferating or quiescent, and what regulates this process? What are the characteristics of the differentiated phenotype of the fibrocyte in the lung? How do the findings from this murine model of lung injury apply to interstitial lung disease in humans?

One of the more exciting aspects of this new pathophysiologic paradigm is the potential impact of bone marrow-derived cells on the development of novel therapeutic approaches for the treatment of pulmonary fibrosis. Investigators in this field are desperately seeking alternative therapeutic targets to slow the progression of pulmonary fibrosis and improve the outcome for patients with this illness (18). Currently, we recognize both the need to intervene early in the natural history of IPF and for novel therapeutic targets to act on proximal events in the fibroproliferative response. We are very hopeful that the recent work presented in the JCI $(2,7)$ will lead to a more comprehensive understanding that ultimately improves the therapeutic options for patients with these progressive and often lethal conditions.

\section{Acknowledgments}

This manuscript was supported by grants from the National Institute of Environmental Health Sciences (ES11375, ES012496, and ES011961), the National Heart, Lung, and Blood Institute (HL67467), and the Department of Veterans' Affairs (Merit Review).

Address correspondence to: David A. Schwartz, Duke University Medical Center, PO Box 2629, Durham, North Carolina 27710, USA. Phone: (919) 668-0380; Fax: (919) 668-0494; E-mail: david.schwartz@duke.edu.

1. Gross, T.J., and Hunninghake, G.W. 2001. Idiopathic pulmonary fibrosis. N. Engl. J. Med. 345:517-525.

2. Phillips, R.J., et al. 2004. Circulating fibrocytes traffic to the lungs in response to CXCL12 and mediate fibrosis. J. Clin. Invest. 114:438-446. doi:10.1172/ JCI200420997.

3. Quan, T.E., Cowper, S., Wu, S.P., Bockenstedt, L.K., and Bucala, R. 2004. Circulating fibrocytes: collagen-secreting cells of the peripheral blood. Int. J. Biochem. Cell Biol. 36:598-606.

4. Bucala, R., Spiegel, L.A., Chesney, J., Hogan, M., and Cerami, A. 1994. Circulating fibrocytes define a new leukocyte subpopulation that mediates tissue repair. Mol. Med. 1:71-81.

5. Aiba, S., Tabata, N., Ohtani, H., and Tagami, H. 1994. CD34+ spindle-shaped cells selectively disappear from the skin lesion of scleroderma. Arch. Dermatol. 130:593-597.

6. Schmidt, M., Sun, G., Stacey, M.A., Mori, L., and Mattoli, S. 2003. Identification of circulating fibrocytes as precursors of bronchial myofibroblasts in asthma. J. Immunol. 171:380-389.

7. Hashimoto, N., Jin, H., Liu, T., Chensue, S.W., and Phan, S.H. 2004. Bone marrow-derived progenitor cells in pulmonary fibrosis. J. Clin. Invest. 113:243-252. doi:10.1172/JCI200418847.

8. Coultas, D.B., Zumwalt, R.E., Black, W.C., and Sobonya, R. 1994. The epidemiology of interstitial lung diseases. Am. J. Respir. Crit. Care Med. 150:967-972.

9. Baumgartner, K.B., Samet, J.M., Stidley, C.A., Colby, T.V., and Waldron, J.A. 1997. Cigarette smoking: A risk factor for idiopathic pulmonary fibrosis. Am. J. Respir. Crit. Care Med. 155:242-248.

10. Hubbard, R., Lewis, S., Richards, K., Johnston, I., and Britton, J. 1996. Occupational exposure to metal or wood dust and aetiology of cryptogenic fibrosing alveolitis. Lancet. 347:284-289.

11. American Thoracic Society. 2000. Idiopathic pulmonary fibrosis: diagnosis and treatment. International consensus statement. Am. J. Resp. Crit. Care Med. 161:646-664.

12. Selikoff, I.J. 1979. Mortality experience of insulation workers in the United States and Canada, 1943-1976. Ann. N. Y. Acad. Sci. 330:91-117.

13. Marshall, R., Puddicombe, A., Cookson, W., and Laurent, G. 2000. Adult familial cryptogenic fibrosing alveolitis in the United Kingdom. Thorax. 55:143-146.

14. Raghu, G., and Hert, R. 1993. Interstitial lung diseases: Genetic predisposition and inherited interstitial lung diseases. Seminars in Respiratory Medicine. 14:323-332.

15. Rossi, G., Szapiel, S., Ferrans, V., and Crystal, R. 1987. Susceptibility to experimental interstitial lung disease is modified by immune- and non-immune related genes. Am. Rev. Respir. Dis. 135:448-455.

16. Ortiz, L.A., et al. 1998. Expression of TNF and the necessity of TNF receptors in bleomycin- induced lung injury in mice. Exp. Lung Res. 24:721-743.

17. Warshamana, G.S., Pociask, D.A., Sime, P., Schwartz, D.A., and Brody, A.R. 2002. Susceptibility to asbestos-induced and transforming growth factor-beta1-induced fibroproliferative lung disease in two strains of mice. Am. J. Respir. Cell Mol. Biol. 27:705-713.

18. Crystal, R.G., et al. 2002. Future research directions in idiopathic pulmonary fibrosis: summary of a National Heart, Lung, and Blood Institute working group. Am. J. Respir. Crit. Care Med. 166:236-246.

19. American Thoracic Society and European Respiratory Society. 2002. American Thoracic Society/European Respiratory Society international multidisciplinary consensus classification of the idiopathic interstitial pneumonias. This joint statement of the American Thoracic Society (ATS), and the European Respiratory Society (ERS) was adopted by the ATS board of directors, June 2001 and by the ERS Executive Committee, June 2001 [review]. Am. J. Respir. Crit. Care Med. 165:277-304. 